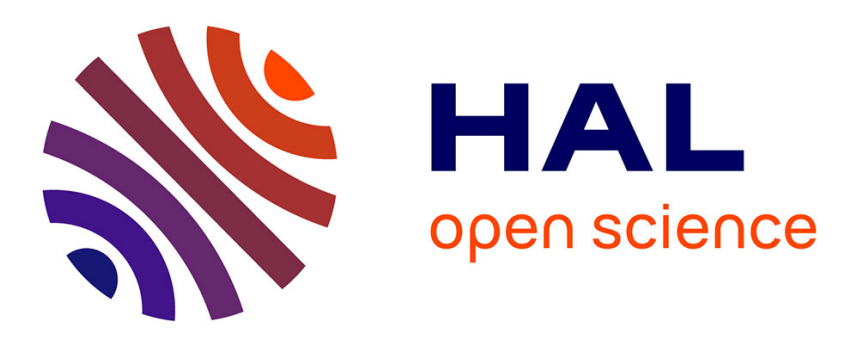

\title{
High Gain Observer for Induction Motor in Presence of Magnetic Hysteresis
}

\author{
Hamid Ouadi, Fouad Giri, Luc Dugard, Abderrahim Elfadili, Philippe \\ Dorléans, Jean-François Massieu
}

\section{- To cite this version:}

Hamid Ouadi, Fouad Giri, Luc Dugard, Abderrahim Elfadili, Philippe Dorléans, et al.. High Gain Observer for Induction Motor in Presence of Magnetic Hysteresis. IFAC WC 2011 - 18th IFAC World Congress, Aug 2011, Milan, Italy. pp.5261-5267. hal-00681423

\section{HAL Id: hal-00681423 \\ https://hal.science/hal-00681423}

Submitted on 21 Mar 2012

HAL is a multi-disciplinary open access archive for the deposit and dissemination of scientific research documents, whether they are published or not. The documents may come from teaching and research institutions in France or abroad, or from public or private research centers.
L'archive ouverte pluridisciplinaire HAL, est destinée au dépôt et à la diffusion de documents scientifiques de niveau recherche, publiés ou non, émanant des établissements d'enseignement et de recherche français ou étrangers, des laboratoires publics ou privés. 


\title{
High Gain Observer for Induction Motor in Presence of Magnetic Hysteresis
}

\author{
H. Ouadi ${ }^{1}$, F. Giri ${ }^{2}$, L. Dugard ${ }^{3}$, A. Elfadili² ${ }^{2}$ Ph-Dorléans ${ }^{2}$, J.F.Massieu ${ }^{2}$ \\ ${ }^{I}$ MACS Lab, University Hassan II, faculty of science, Casablanca, Maroc \\ ${ }^{2}$ GREYC Lab, UMR CNRS, University of Caen, Caen, France \\ ${ }^{3}$ GIPSA Lab, UMR CNRS, INPG, Grenoble, France \\ (Corresponding author: fouad.giri@unicaen.fr)
}

\begin{abstract}
The problem of state estimation in induction motors is considered in this paper. In most previous works, motor observers have been designed using standard models, neglecting the saturation and hysteretic effects in the machine magnetic circuit. As a matter of fact, these effects cannot be ignored especially when considering (speed, torque) control strategies involving wide range flux variations, necessary to meet optimal operation conditions when facing significant load torque changes. In this paper, a high gain observer is designed for induction motors based on a model that accounts for the nonlinear hysteretic nature of the magnetic circuit. The observer provides estimates of the magnetizing current and magnetic variables using available measurements i.e. stator currents and voltages, rotor speed. The observer is formally shown to enjoy nice convergence properties. The theoretical result is confirmed by simulation.
\end{abstract}

\section{INTRODUCTION}

Controlling induction machines requires measurement of electromagnetic and mechanical variables (voltages, currents, flux, speed, position, etc). For some variables (e.g. stator voltages and currents), there exist reliable and not too expensive sensors providing sufficiently accurate measures. This is not the case with other variables such as the rotor flux. Then, observers are resorted to get on-line estimates of all variables which are not accessible to measurement. The first observers (see e.g. Lubineau et al, 1999) were developed based on simplified assumptions namely, linear magnetic characteristics and constant (or slowly varying) rotor speed. Under these assumptions, the model of the induction motor becomes linear and, therefore, observability analysis and observer design may be dealt with, using standard linear theory tools (e.g. pole placement and optimal design, Luenberger and Kalman observers). Interesting contributions came later, proposing nonlinear observers developed without supposing a constant rotor speed (e.g. De Leon et al, 2001). The proposed observers have been designed using different approaches such as high gain, sliding mode and dynamic state feedback. However, even in these contributions, the characteristics of the machine magnetic circuit are still supposed to be linear. The point is that this assumption is only valid when the machine remains all time in the vicinity of its nominal flux value. But, a constant-flux operation mode cannot be optimal when wide range speed variations are needed with a machine facing large load torque changes. To achieve high observation performances, regardless the machine operation mode, the observer design should be based on a model that accounts for the nonlinearity of the machine magnetic circuit. This nonlinearity is characterized both by saturation and hysteresis. Few previous works have attempted to deal with the machine magnetic circuit nonlinearity in observers design (Krzeminski et al., 1993), (Ouadi et al.,
2005). Furthermore, the models based upon in these works only accounted for the magnetic circuit saturation (neglecting the hysteretic effect).

In this paper, a new state observer is proposed for estimating the rotor flux (and stator currents) in induction motors facing large speed and load torque variations. It is designed by the high gain technique, using a recently developed model that properly accounts for hysteresis in the magnetic circuit (Ouadi et al., 2010). In this model, the hysteresis effect is captured through the Coleman-Hodgdon model that proved to be quite convenient for hysteresis modelling in ferromagnetic materials (Coleman \& Hodgdon, 1987; Macki et al., 1993). In the $\alpha \beta$-coordinates, the new model turns out to be a $7^{\text {th }}$ order, there where the standard models are $5^{\text {th }}$ orders. The two new state variables are the magnetizing current components. For the purpose of observer design, a Park model transformation is first performed, yielding a more tractable model fitting the required structure for the high-gain observer design technique to be applicable. Therefore, the obtained observer is formally proved, under standard assumptions, to be globally asymptotic convergent. This result is confirmed by a numerical simulation involving significant changes of the speed and load torque, making the machine operate both in linear and nonlinear parts of its magnetic characteristic. The observer thus developed can be used as an element of a more global control strategy seeking (in addition to speed regulation) further objectives e.g. power efficiency improvement, flux optimisation, power factor correction, etc. These cannot be achieved with observers obtained from standard models because these are only representative of the machine within a limited flux range.

The paper is organised as follows: the model of the induction motor is presented in Section 2; the high-gain observer is designed and analysed in Section 3, the observer 
performances are illustrated by simulation in Section 4; a conclusion and reference list end the paper.

\section{INDUCTION MOTOR MODELLING}

A model that accounts for the magnetic circuit hysteresis has recently been developed and validated in (Ouadi et al., 2010). The hysteresis phenomenon (Fig. 1) is captured through the Coleman-Hodgdon (C-H) model (Coleman \& Hodgdon, 1987), (Macki et al., 1993). The complete machine model has been given the following state-space representation:

$$
\begin{aligned}
\frac{d i_{s \alpha}}{d t}= & -\frac{R_{s}+R_{r}}{L s} i_{s \alpha}+\frac{R_{r}}{L s} i_{\mu \alpha}+p \Omega \frac{\phi_{r \beta}}{L_{s}}+\frac{1}{L_{s}} u_{s \alpha} \\
\frac{d i_{s \beta}}{d t}= & -\frac{R_{s}+R_{r}}{L s} i_{s \beta}+\frac{R_{r}}{L s} i_{\mu \beta}-p \Omega \frac{\phi_{r \alpha}}{L_{s}}+\frac{1}{L_{s}} u_{s \beta} \\
\frac{d \phi_{r \alpha}}{d t}= & -R_{r} \cdot i_{\mu \alpha}+R_{r} \cdot i_{s \alpha}-p \Omega \phi_{r \beta} \\
\frac{d \phi_{r \beta}}{d t}= & -R_{r} \cdot i_{\mu \beta}+R_{r} \cdot i_{s \beta}+p \Omega \phi_{r \alpha} \\
\frac{d \Omega}{d t}= & \left.\frac{p}{J}\left(\phi_{r \alpha} i_{s \beta}-\phi_{r \beta} i_{s \alpha}\right)\right)-\frac{T_{L}}{J}-\frac{f}{J} \Omega \\
\frac{d i_{\mu \alpha}}{d t}= & \frac{1}{6}\left(4 h_{1}+h_{2}+h_{3}\right)\left(-R_{r} \cdot i_{\mu \alpha}+R_{r} \cdot i_{s \alpha}-\omega \phi_{r \beta}\right) \\
& -\frac{\sqrt{3}}{6}\left(h_{2}-h_{3}\right)\left(-R_{r} . i_{\mu \beta}+R_{r} \cdot i_{s \beta}+\omega \phi_{r \alpha}\right) \\
\frac{d i_{\mu \beta}}{d t}= & -\frac{\sqrt{3}}{6}\left(h_{2}-h_{3}\right)\left(-R_{r} \cdot i_{\mu \alpha}+R_{r} . i_{s \alpha}-\omega \phi_{r \beta}\right) \\
& +\frac{1}{2}\left(h_{2}+h_{3}\right)\left(-R_{r} \cdot i_{\mu \beta}+R_{r} . i_{s \beta}+\omega \phi_{r \alpha}\right)
\end{aligned}
$$

where:

- $\left(\phi_{r \alpha}, \phi_{r \beta}\right),\left(i_{s \alpha}, i_{s \beta}\right),\left(i_{\mu \alpha}, i_{\mu \beta}\right)$ and $u=\left[u_{s \alpha}, u_{s \beta}\right]^{T}$ are the $\alpha \beta$-components of the rotor flux, stator current, magnetizing current and stator voltage, respectively;

- $\Omega$ is the motor speed;

- $R_{s}, R_{r}$ denote the stator and rotor resistances;

- $T_{L}$ is the load torque;

- $p$ designates the number of pole pairs;

- $L_{s}$ is the equivalent inductance (of both stator and rotor);

- The $h_{k}$ 's are functions of the magnetizing currents:

$$
h_{k}=\left(\alpha \operatorname{sign}\left(i_{\mu k}\right)\left[f_{c}\left(i_{\mu k}\right)-\phi_{r k}\right]+g_{c}\left(i_{\mu k}\right)\right)(k=1,2,3)
$$

- $f_{c}($.$) and \mathrm{g}_{\mathrm{c}}($.$) are two functions:$

$$
\begin{aligned}
& f\left(i_{\mu}\right)=c \tanh \left(i_{\mu}\right)+a i_{\mu} \\
& g\left(i_{\mu}\right)=f^{\prime}\left(i_{\mu}\right)\left(1-b e^{-\left|i_{\mu}\right|}\right)
\end{aligned}
$$

The five first equations (1-5) are standard, except for the dependence, now made explicit, on the magnetizing currents $\left(i_{\mu \alpha}, i_{\mu \beta}\right)$. The remaining equations (6-7) account for the hysteresis effect in the magnetic circuit. They involve the functions $f_{c}($.$) and \mathrm{g}_{\mathrm{c}}($.$) which are a part of the \mathrm{C}-\mathrm{H}$ hysteresis model (Ouadi et al., 2010).

All the parameters can be identified via specific experimental measurements on real-life machine. This also concerns the hysteresis parameters $(a, b, c, \alpha)$ that can be obtained from the machine magnetic characteristics (Fig. 1), (Ouadi et al., 2010).

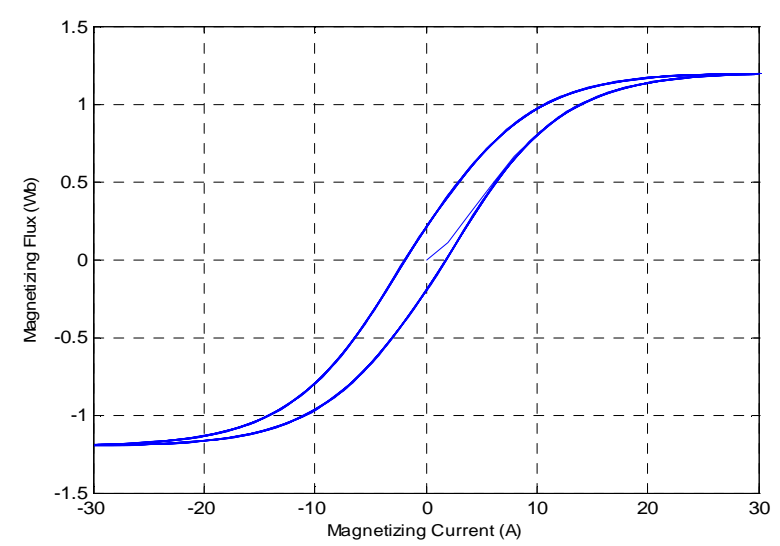

Fig 1: Hysteresis curve given by $\mathrm{C}-\mathrm{H}$ model.

\section{STATE OBSERVER DESIGN}

\subsection{Motor model reformulation}

The observer is resorted to estimate online the machine state variables that are not accessible to measurements i.e. the magnetizing current and the flux. It will be obtained from the transformed model (1-7) using the high gain design technique, e.g. (Besançon, 2007). To reduce the number of equations, the Park transformation is applied to the model (17 ). The obtained $d q$-coordinates model is oriented along the magnetizing current. Doing so, one gets the following form:

$$
\begin{aligned}
& \dot{x}=F(x) x+G(u, x) \\
& y=C x
\end{aligned}
$$

with:

$$
x=\left[\begin{array}{l}
x^{1} \\
x^{2}
\end{array}\right]
$$

where:

$$
\begin{aligned}
& x^{1}=\left[\begin{array}{lll}
i_{s d} & i_{s q} & \Omega
\end{array}\right]^{T}=\left[\begin{array}{lll}
x_{1} & x_{2} & x_{3}
\end{array}\right]^{T} \\
& x^{2}=\left[\begin{array}{lll}
\phi_{r d} & \phi_{r q} & i_{\mu d}
\end{array}\right]^{T}=\left[\begin{array}{lll}
x_{4} & x_{5} & x_{6}
\end{array}\right]^{T} \\
& u=\left[\begin{array}{lll}
u_{s d} & u_{s q} & \omega_{s}
\end{array}\right]^{T}, \text { control input } \\
& y=\left[\begin{array}{lll}
i_{s d} & i_{s q} & \Omega
\end{array}\right]^{T}, \text { output vector }
\end{aligned}
$$

$G(u, x)=\left[\begin{array}{c}g^{1}\left(u, x^{1}\right) \\ g^{2}(u, x)\end{array}\right]$

$g^{1}\left(u, x^{1}\right)=\left[\begin{array}{l}-\frac{R_{s}+R_{r}}{L s} i_{s d}+\omega_{s} i_{s q}+\frac{1}{L_{s}} u_{s d} \\ -\frac{R_{s}+R_{r}}{L s} i_{s q}-\omega_{s} i_{s d}+\frac{1}{L_{s}} u_{s q} \\ \frac{T_{L}}{J}-\frac{f}{J} \Omega\end{array}\right]$

$g^{2}(x, u)=\left[\begin{array}{l}-R_{r} i_{\mu d}+R_{r} i_{s d}+\left(\omega_{s}-p \Omega\right) \phi_{r q} \\ R_{r} i_{s q}-\left(\omega_{s}-p \Omega\right) \phi_{r d} \\ A_{11}\left(-R_{r} \cdot i_{\mu d}+R_{r} \cdot i_{s d}+p \Omega \phi_{r q}\right)+A_{12}\left(R_{r} \cdot i_{s q}-p \Omega \phi_{r d}\right)\end{array}\right]$ 
where:

$$
\begin{aligned}
& A_{11}=H_{11} \cos ^{2}\left(\theta_{s}\right)+H_{12} \sin \left(2 \theta_{s}\right)+H_{22} \sin ^{2}\left(\theta_{s}\right) \\
& A_{12}=-0.5 H_{11} \sin \left(2 \theta_{s}\right)+H_{12} \cos \left(2 \theta_{s}\right)+0.5 H_{22} \sin \left(2 \theta_{s}\right) \\
& A_{22}=H_{11} \sin ^{2}\left(\theta_{s}\right)-H_{12} \sin \left(2 \theta_{s}\right)+H_{22} \cos ^{2}\left(\theta_{s}\right) \\
& \text { where }\left[\begin{array}{ll}
H_{11} & H_{12} \\
H_{12} & H_{22}
\end{array}\right]=\left[\begin{array}{cc}
\frac{1}{6}\left(4 h_{1}+h_{2}+h_{3}\right) & \frac{\sqrt{3}}{6}\left(h_{3}-h_{2}\right) \\
\frac{\sqrt{3}}{6}\left(h_{3}-h_{2}\right) & \frac{1}{2}\left(h_{3}+h_{2}\right)
\end{array}\right] \\
& F(x)=\left[\begin{array}{cc}
0_{3} & F^{1}\left(x^{1}\right) \\
0_{3} & 0_{3}
\end{array}\right] \\
& F^{1}\left(x^{1}\right)=\left[\begin{array}{ccc}
0 & \frac{p \Omega}{L_{s}} & +\frac{R_{r}}{L_{s}} \\
-\frac{p \Omega}{L_{s}} & 0 & 0 \\
\frac{p}{J} i_{s q} & -\frac{p}{J} i_{s d} & 0
\end{array}\right] \\
& C=\left[\begin{array}{llllll}
1 & 1 & 1 & 0 & 0 & 0
\end{array}\right]
\end{aligned}
$$

The notation $0_{3}$ stands for the $3 \times 3$ zero matrix. Note that, the above required dq-frame orientation can be ensured by making a suitable choice of the input stator frequency $\omega_{s}$. Indeed, by letting $i_{\mu q}=i_{\mu q}=0$, it follows that $\omega_{s}$ must satisfy the following condition:

$$
\omega_{s}=\frac{1}{i_{\mu d}}\left(R_{r} A_{12} .\left(i_{s d}-i_{\mu d}\right)+R_{r} A_{22} . i_{s q}+\omega\left(-A_{22} \phi_{r d}+A_{12} \phi_{r q}\right)\right)
$$

In the rest of the study, the condition (19) is supposed to be fulfilled.

The observer synthesis and analysis necessitate the following assumption:

A1. The state vector $x$ and the control vector $u$ are bounded.

Remark 1. The above assumptions are natural in the present open-loop control context. Furthermore, it is readily seen from (17) that the function $F^{1}\left(x^{1}\right)$ is linear and so is Lipschitz

\subsection{Model transformation}

Consider the following state transformation:

$$
\begin{aligned}
& T: I R^{6} \rightarrow I^{6} \\
& x=\left[\begin{array}{l}
x^{1} \\
x^{2}
\end{array}\right] \rightarrow z=T(x)=\left[\begin{array}{c}
x^{1} \\
F^{1}\left(x^{1}\right) x^{2}
\end{array}\right]
\end{aligned}
$$

For convenience, the transformation is given the following compact form:

$$
z=T(x)=\Lambda(x) x
$$

with

$$
\Lambda(x)=\left[\begin{array}{cc}
I_{3} & 0_{3} \\
0_{3} & F^{1}\left(x^{1}\right)
\end{array}\right]
$$

where $I_{3}$ denotes the $3 \times 3$ identity matrix.

Remark 2. a) Using Assumption A.1, one gets from (20) that the new state vector $\mathrm{z}$ is also bounded. b) Also, using A1, it follows that $T($.$) is Lipschitz.$ Furthermore, it is clear that the transformation $T($.$) is$ invertible

c) As $F^{1}\left(x^{1}\right)$ is Lipschitz (Remark 1), equation (22) implies that $\Lambda$ is in turn Lipschitz

Using (20) and (11), it is readily shown that:

$\dot{z}=\frac{\partial T}{\partial x}(x) \dot{x}(t)=\frac{\partial T}{\partial x}(x)(F(x) x+G(u, x))$

which, together with (21), yields:

$\dot{z}=\Lambda(x) F(x) x+\left(\frac{\partial T}{\partial x}(x)-\Lambda(x)\right) F(x) x+\frac{\partial T}{\partial x}(x) G(u, x)$

Using the notation

$A=\left[\begin{array}{ll}0_{3} & I_{3} \\ 0_{3} & 0_{3}\end{array}\right]$

it can be easily checked, using (22) and (17), that:

$\Lambda(x) F(x)=A \Lambda(x)$

or, equivalently:

$F(x)=\Lambda^{-1}(x) A \Lambda(x)$

It is easily seen from (22) that:

$\Lambda^{-1}(x)=\left[\begin{array}{cc}I_{3} & 0_{3} \\ 0_{3} & \left(F^{1}\left(x^{1}\right)\right)^{-1}\end{array}\right]$

Furthermore, it can be checked using (17) that:

$$
\left(F^{1}\left(x^{1}\right)\right)^{-1}=\left[\begin{array}{ccc}
0 & \frac{-L_{s}}{p \Omega} & 0 \\
0 & 0 & \frac{-L_{s} J}{p i_{s d}} \\
\frac{-L_{s}}{R_{r}} & \frac{-L_{s} i_{s q}}{R_{r} i_{s d}} & \frac{-L_{s} p \Omega J}{p R_{r} i_{s d}}
\end{array}\right]
$$

Remark 3. It is well known that, in normal operation conditions, the machine stator current can never be in phase or quadrature phase with the magnetizing current (Leonard, 2001). Bearing in mind the $(d, q)$-frame orientation (along the current $i_{\mu d}$ ), it follows that the stator current components are necessarily nonzero $\left(i_{s d} \neq 0, i_{s q} \neq 0\right)$. Then, $\left(F^{1}\left(x^{1}\right)\right)^{-1}$ involves no singularity whenever the machine is rotating i.e. for a non zero rotor speed

Let us introduce the following notations:

$$
\begin{aligned}
& \Theta(z)=\left(\frac{\partial T}{\partial x}\left(T_{i n v}(z)\right) \Lambda^{-1}\left(T_{i n v}(z)\right)-I_{6}\right) A z \\
& \varphi(u, z)=\frac{\partial T}{\partial x}\left(T_{i n v}(z)\right) G(u, x)
\end{aligned}
$$

Introducing (27) in (24) leads to the new state-space representation of the induction machine:

$\dot{z}=A z+\Theta(z)+\varphi(u, z)$ 
Note that this also accounts, in the $\mathrm{z}$-coordinates, for the magnetic circuit hysteresis.

Remark 4. a) From (22) one gets:

$\frac{\partial T}{\partial x}=\left[\begin{array}{cc}I_{3} & 0_{3} \\ A_{1}(x) & F^{1}\left(x^{1}\right)\end{array}\right]$ with $A_{1}(x)=\left[\begin{array}{ccc}0 & 0 & \frac{p}{L_{s}} x_{5} \\ 0 & 0 & -\frac{p}{L_{s}} x_{4} \\ -\frac{p}{J} x_{5} & \frac{p}{J} x_{4} & 0\end{array}\right]$

From Remark 1 and the fact that the last matrix is linear in state variables, it follows from (33) that $\frac{\partial T}{\partial x}$ is Lipschitz.

Using (30), (33), (28) and (25), one obtains:

$$
\Theta(z)=\left[\begin{array}{ll}
0_{3} & \\
A_{1}(x) & {\left[\begin{array}{l}
z_{4} \\
z_{5} \\
z_{6}
\end{array}\right]}
\end{array}\right]=\left[\begin{array}{l}
0_{3} \\
\frac{p}{L_{s}} \phi_{r q} z_{6} \\
-\frac{p}{L_{s}} \phi_{r d} z_{6} \\
-\frac{p}{J} \phi_{r q} z_{4}+\frac{p}{J} \phi_{r d} z_{5}
\end{array}\right]
$$

Referring again to Assumption A1 and to the considerations described in Remark 3, the function $\Theta(z)$ turns out to be Lipschitz whenever the machine is rotating (non zero speed).

c) Similarly, using the fact that:

$$
z^{1}=\left[\begin{array}{lll}
z_{1} & z_{2} & z_{3}
\end{array}\right]^{T}=\left[\begin{array}{lll}
x_{1} & x_{2} & x_{3}
\end{array}\right]^{T} .
$$

it follows from (31), (34) and (15) that:

$$
\varphi(z, u)=\left[\begin{array}{cc}
I_{3} & 0_{3} \\
A_{1}(x) & F^{1}\left(x^{1}\right)
\end{array}\right]\left[\begin{array}{l}
g^{1}\left(x^{1}, u\right) \\
g^{2}\left(x^{1}, x^{2}, u\right)
\end{array}\right]
$$

From (34) and (15), it is readily seen that $A_{1}(x)$ and $g^{2}(x, u)$ are bounded, because $x$ and $u$ are so. Furthermore, the functions $g^{1}\left(z^{1}, u\right)$ and $F^{1}\left(z^{1}\right)$ are clearly Lipschitz due their linearity. Then, equation (35) implies that that $\varphi(z, u)$ is in turn Lipschitz

\subsection{Machine observer in z-coordinates}

Let $\Delta_{\theta}$ be the block diagonal matrix defined by:

$\Delta_{\theta}=\operatorname{diag}\left\lfloor I_{3} \quad \frac{1}{\theta} I_{3}\right\rfloor$

where $\theta$ is any real positive constant. Let $S$ be the solution of the algebraic Lyapunov equation:

$$
S+A^{T} S+S A-C^{T} C=0
$$

where $A$ and $C$ are respectively given by equations (25) and (18). It is shown in many works (e.g. Besançon, 2007) that the solution $S$ of (37) is unique, symmetric, positive definite and satisfies:

$$
S^{-1} C^{T}=\left[\begin{array}{l}
I_{3} \\
0_{3}
\end{array}\right]
$$

With the above notations, a candidate observer for the system (32) is the following:

$$
\dot{\hat{z}}=A \hat{z}+\Theta(\hat{z})+\varphi(u, \hat{z})-\theta \Delta_{\theta}^{-1} S^{-1} C^{T}(C \hat{z}-y)
$$

$$
-\theta\left(\frac{\partial T}{\partial x}\left(T_{i n v}(\hat{z})\right) \Lambda^{-1}\left(T_{i n v}(\hat{z})\right)-I_{6}\right) \Delta_{\theta}^{1} S^{-1} C^{T}(C \hat{z}-y)
$$

Proposition 1. The state observer (39), when applied to the nonlinear system (32) (where all signals $(u, z, y)$ are bounded), is globally exponentially convergent. More specifically, there exists a $\theta_{0}>0$ such that for all $\theta>\theta_{0}$, there is a $\sigma_{\theta}>0$ so that:

$\|\hat{z}(t)-z(t)\| \leq \kappa_{S} \theta \exp \left(-\sigma_{\theta} t\right)\|\hat{z}(0)-z(0)\|$

whatever $\hat{z}(0) \in I R^{6}$ where $\kappa_{S}$ denotes the condition number of the matrix $S$. Furthermore, one has:

$\lim _{\theta \rightarrow+\infty} \sigma_{\theta}=+\infty$

Proof. Let $e(t)=\hat{z}(t)-z(t)$. Then,

$$
\begin{aligned}
\dot{e} & =\left(A-\theta \Delta_{\theta}^{-1} S^{-1} C^{T} C\right) e+(\Theta(\hat{z})-\Theta(z)) \\
& +(\varphi(u, \hat{z})-\varphi(u, z))-\theta \Gamma(\hat{z}) \Delta_{\theta}^{-1} S^{-1} C^{T} C e
\end{aligned}
$$

with

$$
\Gamma(\hat{z})=\frac{\partial T}{\partial x}\left(T_{i n v}(\hat{z})\right) \Lambda^{-1}\left(T_{i n v}(\hat{z})\right)-I_{6}
$$

Substituting (33), (22) and (28) in (42), one obtains from (41):

$$
\Gamma(\hat{z})=\left[\begin{array}{cc}
0_{3} & 0_{3} \\
A_{1}\left(T_{\text {inv }}(\hat{z})\right) & 0_{3}
\end{array}\right]
$$

It is easily checked that:

$\theta \Delta_{\theta}^{-1} A \Delta_{\theta}=A$ and $C^{T} C \Delta_{\theta}=C^{T} C$

Hence,

$$
\begin{aligned}
\dot{e}=\theta & \Delta_{\theta}^{-1}\left(A-S^{-1} C^{T} C\right) \Delta_{\theta} e+(\Theta(\hat{z})-\Theta(z)) \\
& +(\varphi(u, \hat{z})-\varphi(u, z))-\theta \Gamma(\hat{z}) \Delta_{\theta}^{-1} S^{-1} C^{T} C e
\end{aligned}
$$

Introduce the variable change:

$$
\bar{e}=\Delta_{\theta} e
$$

It follows using (41) that:

$$
\begin{aligned}
\dot{\bar{e}} & =\theta\left(A-S^{-1} C^{T} C\right) \bar{e}+\Delta_{\theta}(\Theta(\hat{z})-\Theta(z)) \\
& +\Delta_{\theta}(\varphi(u, \hat{z})-\varphi(u, z))-\theta \Delta_{\theta} \Gamma(\hat{z}) \Delta_{\theta}^{-1} S^{-1} C^{T} C e
\end{aligned}
$$

Consider the Lyapunov function candidate $V(\bar{e})=\bar{e}^{T} S \bar{e}$. Its time derivative along the trajectory of (46) is:

$$
\begin{aligned}
\dot{V} & =2 \bar{e}^{T} S \dot{\bar{e}} \\
= & -\theta V-\bar{e}^{T} C^{T} C \bar{e}+2 \bar{e}^{T} S \Delta_{\theta}(\Theta(\hat{z})-\Theta(z)) \\
& +2 \bar{e}^{T} S \Delta_{\theta}(\varphi(u, \hat{z})-\varphi(u, z))-2 \theta \bar{e}^{T} S \Delta_{\theta} \Gamma(\hat{z}) \Delta_{\theta}^{-1} S^{-1} C^{T} C e
\end{aligned}
$$

which together with (38) implies:

$$
\begin{aligned}
\dot{V} \leq & -\theta V+2\|\bar{e}\|\left\|\Delta_{\theta}(\Theta(\hat{z})-\Theta(z))\right\| \\
+ & 2\|\bar{e}\|\left\|\Delta_{\theta}(\varphi(u, \hat{z})-\varphi(u, z))\right\| \\
& -2 \theta\left\|\bar{e}^{T}\right\|\left\|\Delta_{\theta} \Gamma(\hat{z}) \Delta_{\theta}^{-1}\right\|\left\|S^{-1} C^{T} C e\right\|
\end{aligned}
$$

Letting $\theta \geq 1$, it follows using the triangular structure and Lipschitz nature of $\Theta, \varphi$ and $\partial T / \partial x$ (Remark 3) one gets:

$\left\|\Delta_{\theta}(\Theta(\hat{z})-\Theta(z))\right\| \leq \rho_{1}\|\bar{e}\|$

$\left\|\Delta_{\theta}(\varphi(u, \hat{z})-\varphi(u, z))\right\| \leq \rho_{2}\|\bar{e}\|$ 
for some positive constants $\rho_{1}$ and $\rho_{2}$ independent on $\theta$. Furthermore, the Lipschitz property of $\partial T / \partial x$ and $\Lambda$ (Remarks 3 and 4) and the boundedness of all signals imply that $\Gamma$ is bounded. Moreover, since $\Gamma$ is lower triangular with zeros on the main diagonal (43), one gets:

$\left\|\theta \Delta_{\theta} \Gamma(\hat{z}) \Delta_{\theta}^{-1}\right\| \leq \rho_{3} \quad$ for $\quad \theta \geq 1$

for some constant $\rho_{3}>0$ independent on $\theta$. In view of (49)(51), inequality (48) yields:

$$
\begin{aligned}
\dot{V} & \leq-\theta V+2\left(\rho_{1}+\rho_{2}+\rho_{3}\right)\|\bar{e}\|^{2} \\
& \leq-\theta V+c_{1} V \quad(\text { using (42)) }
\end{aligned}
$$

It readily follows from $(52)$ that:

$$
V(t) \leq \exp \left[-\left(\theta-c_{1}\right) t\right] V(0)
$$

Now, letting $\theta_{0}=\max \left\{1, c_{1}\right\}$ and $\theta>\theta_{0}$, one obtains from (53), using the fact that $V(\bar{e})=\bar{e}^{T} S \bar{e}$ :

$\|\bar{e}(t)\| \leq \kappa_{S} \exp \left[-\frac{\left(\theta-c_{1}\right) t}{2}\right]\|\bar{e}(0)\|$

On the other hand, for $\theta>\theta_{0}$, it follows using (45) and (36):

$$
\|\bar{e}\| \leq\|e\| \leq \theta\|\bar{e}\|
$$

Combining (54) and (55), one gets:

$\|e(t)\| \leq \kappa_{S} \theta \exp \left[-\frac{\left(\theta-c_{1}\right) t}{2}\right]\|e(0)\|$

which ends the proof of Theorem

\subsection{Machine observer in $x$-coordinates}

The transformation (20) suggests that $x$-estimates can be obtained from $z$-estimates by solving the following equation: $T(\hat{x})=\hat{z}$.

Differentiating both sides of (57) with respect to time yields:

$$
\frac{\partial T(\hat{x})}{\partial \hat{x}} \dot{\hat{x}}=\dot{\hat{z}}
$$

Using (39) and (30), it follows from (58):

$$
\begin{aligned}
\dot{\hat{x}} & =\left(\frac{\partial T(\hat{x})}{\partial \hat{x}}\right)^{-1}\left[A T(\hat{x})+\left(\frac{\partial T}{\partial x}(\hat{x}) \Lambda^{-1}(\hat{x})-I_{6}\right) A T(\hat{x})\right. \\
& +\varphi(u, \hat{z})-\theta \Delta_{\theta}^{-1} S^{-1} C^{T}(C \hat{z}-y)
\end{aligned}
$$

which, together with (31) and (21), gives:

$$
\begin{aligned}
& \dot{\hat{x}}=\Lambda^{-1}(\hat{x}) A \Lambda(\hat{x}) \hat{x}-\left(\frac{\partial T}{\partial x}(\hat{x})\right)^{-1}\left(\frac{\partial T}{\partial x}(\hat{x})\right) G(u, \hat{x}) \\
&-\theta\left(\frac{\partial T}{\partial x}(\hat{x})\right)^{-1} \Delta_{\theta}^{-1} S^{-1} C^{T}(C \hat{z}-y) \\
&-\theta\left(\frac{\partial T}{\partial x}(\hat{x})\right)^{-1}\left(\frac{\partial T}{\partial x}(\hat{x}) \Lambda^{-1}(\hat{x})-I_{6}\right) \Delta_{\theta}^{-1} S^{-1} C^{T}(C \hat{z}-y) \\
&\left.-\theta\left(\frac{\partial T}{\partial x}(\hat{x}) \Lambda^{-1}(\hat{x})-I_{6}\right) \Delta_{\theta}^{-1} S^{-1} C^{T}(C \hat{z}-y)\right]
\end{aligned}
$$

Using (27), equation (60) simplifies to:

$$
\begin{aligned}
\dot{\hat{x}} & =F(\hat{x}) \hat{x}-G(u, \hat{x}) \\
& -\theta\left(\frac{\partial T}{\partial x}(\hat{x})\right)^{-1}\left(I_{6}+\frac{\partial T}{\partial x}(\hat{x}) \Lambda^{-1}(\hat{x})-I_{6}\right) \Delta_{\theta}^{-1} S^{-1} C^{T}(C \hat{z}-y)
\end{aligned}
$$

which gives the final form of the $x$-coordinate observer:

$$
\dot{\hat{x}}=F(\hat{x}) \hat{x}-G(u, \hat{x})-\theta \Lambda^{-1}(\hat{x}) \Delta_{\theta}^{-1} S^{-1} C^{T}(C \hat{x}-y)
$$

Theorem 1. The state observer (61), when applied to the induction machine model (11) subject to Assumptions (A1A2), is globally exponentially convergent. More specifically, there exist real constants $\lambda, \theta_{0}>0$ such that for any real $\theta>\theta_{0}$ there is a real $\sigma_{\theta}>0$ so that:

$\|\hat{x}(t)-x(t)\| \leq \lambda \theta \exp \left[-\sigma_{\theta} t\right]\|\hat{x}(0)-x(0)\|$

whatever $\hat{x}(0)$; where $x(t)$ denotes the unknown trajectory of (11) associated to the input signal $u(t)$. Furthermore:

$\lim _{\theta \rightarrow+\infty} \sigma_{\theta}=+\infty$

Proof. Introduce the estimation error $\tilde{x}(t)=\hat{x}(t)-x(t)$. From (20) and (57), one has:

$\tilde{x}(t)=T_{i n v}(\hat{z})-T_{i n v}(z)$

As $T_{i n v}$ (.) is Lipschitz, it follows from (63) that:

$|\tilde{x}(t)| \leq \delta|\hat{z}-z|$

where $\delta$ denotes the Lipschitz constant of $T_{i n v}($.$) . Using$ Proposition 1, one gets:

$\|\tilde{x}\| \leq \delta \kappa_{S} \theta \exp \left[-\frac{\left(\theta-c_{1}\right) t}{2}\right]\|\tilde{z}(0)\|$

This establishes Theorem 1, using the fact that $z=T(x)$ is a Lipschitz function (Remark 2, part b)

\section{SIMULATION}

The induction machine characteristics are described in (Ouadi et al., 2005). The functions $f_{c}$ and $g_{c}$, that characterize the model hysteresis part (8-10), are given the following values determined in (Ouadi et al., 2010):

$$
a=0.01, b=0.601, c=0.995, d=1.2 \text { and } \alpha=0.4
$$

The simulation protocol is conceived in a way that makes the machine operates around different points of its magnetic characteristic, different rotor speeds and different load torque values. Doing so, the hysteresis effect on the machine behavior is meaningful. To this end, the stator voltage and load torque are let to be varying as shown by Fig. 2. The machine is open-loop controlled using a sinusoidal stator voltage input. The remaining simulation details are the following: the observer gain is $\theta=100$ and the initial conditions of the observer and the true system model are quite different $\left(\hat{\Phi}_{r}(0)=0.75(w b), \Phi_{r}(0)=0.15(w b)\right)$. Figs. 3 to 4 show that the state estimates converge to their true values after a transient period that lasts less than 0.5 second. 

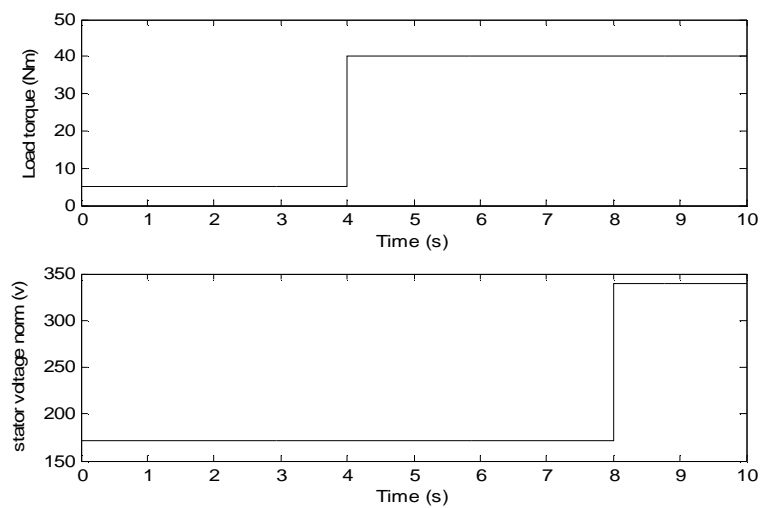

Fig. 2: Upper : Load torque (Nm) Lower : Stator voltage norm (v)
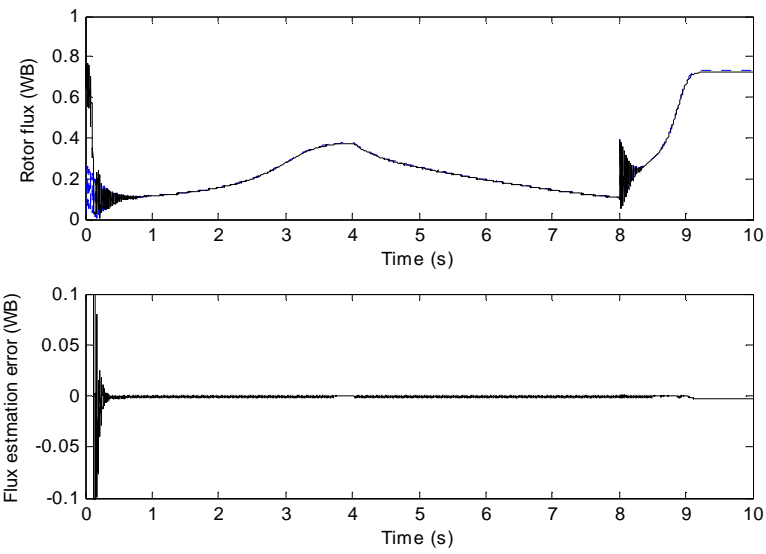

Fig 3: Rotor flux norm (Wb) estimation. Upper: real and observed flux norm; lower: estimation error
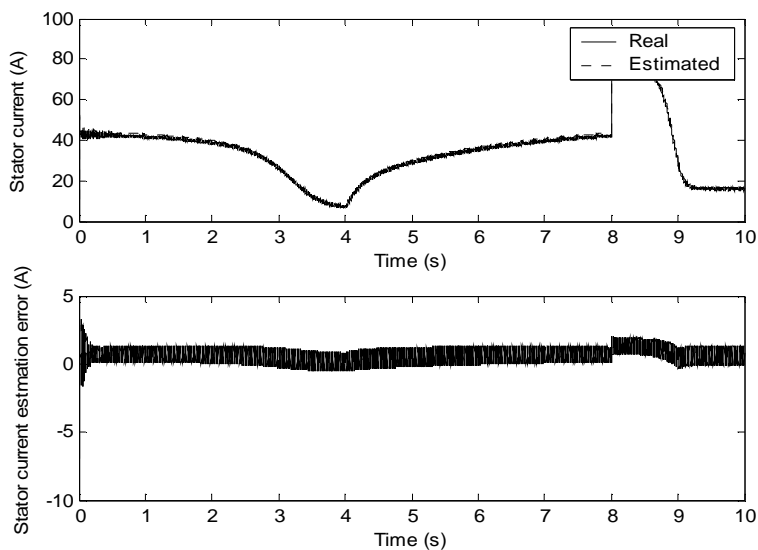

Fig. 4: Stator current norm estimation. Upper: measured and estimated current norm; lower: estimation error

\section{CONCLUSION}

The problem of state observation is addressed for induction machines. The originality lies in the fact that the problem is dealt with, based a model that accounts for the magnetic circuit hysteresis. A state observer is thus developed using the high-gain design technique and it is formally shown to be exponentially convergent. This result is confirmed by simulation using the numerical parameter values of a $7.5 \mathrm{~kW}$ induction motor.

\section{REFERENCES}

Besançon G., (2007). 'Nonlinear Observers and Applications'. Springer Berlin / Heidelberg.

Coleman B. D., M. L. Hodgdon, (1987). "On a class of constitutive relations for ferromagnetic hysteresis'. Arch. Rational Mech. Anal., pp. 375-396.

De Leon J., Alvarez-Leal J. G., Castro-Linares R., and Alvarez-Gallegos J. (2001). "Control of a flexible joint robot manipulator via a nonlinear control-observer scheme". International Journal of Control, 74(3): 290302 .

Heinemann G. and W. Leonhard (1990). "Self-tuning fieldoriented control of an induction motor drive". International Power Electronics Conference, Tokyo, Japan.

Khalil H. (2003). “Nonlinear systems”. Prentice-Hall.

Kim K., Ortega R., Charara A. and Vilain J. P. (1997). "Theoretical and experimental comparison of two nonlinear controller for induction motors". IEEE Transactions on Control Systems Technology, vol. 5, no 3, pp. 338-348.

Krzeminski Z, A. Jaderko (1993). "Main magnetic path saturation effect in observer system of the induction motor". IEEE International Symposium on Industrial Electronics, Budapest, Hungry, 753 - 758.

Leonard W., (2001). "Control of Electrical Drives". Springer.

Lubineau D., J.M. Dion, L. Dugard, and D. Roye. (2000). "Design of an advanced nonlinear controller for induction motor and experimental validation on an industrial benchmark", EPJ Applied Physics, vol. 9, pp. 165-175.

Macki J. W., P. Nistri, P. Zecca, (1993) "Mathematical models for hysteresis". SIAM Review, vol. 35, no. 1, pp. 94-123.

Ouadi, H., F. Giri, J. De Leon-Morales, and L. Dugard (2005) "High gain observer design for induction motor with non linear magnetic characteristic". IFAC World Congress, Prague, Czech Republic.

Ouadi H., Giri F., Elfadili A., Dorléans P. and Massieu J.F. (2010). "Induction Machine Control in Presence of Magnetic hysteresis: Modelling and Speed reference tracking" IFAC International Workshop on Adaptation and Learning in Control and Signal Processing ALCOSP, 26-28, Antalya, Turkey. 